\title{
OPEN
}
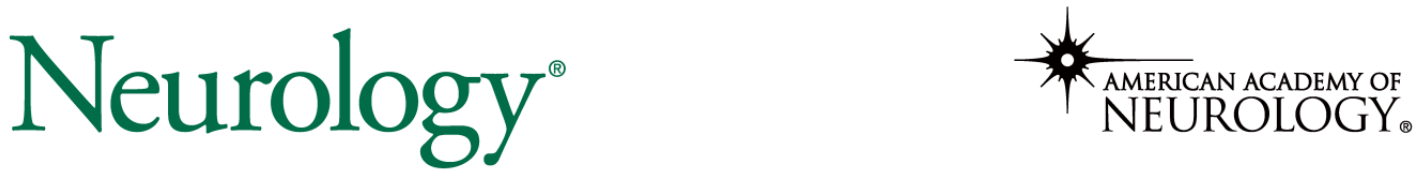

The most widely read and highly cited peer-reviewed neurology journal

The Official Journal of the American Academy of Neurology

Neurology Publish Ahead of Print DOI: 10.1212/WNL.0000000000013123

\section{Amyotrophic Lateral Sclerosis Frontotemporal Dementia: Shared and Divergent Neural Correlates Across the Clinical Spectrum}

\author{
Author(s): \\ Camilla Cividini, MSc ${ }^{1,2}$; Silvia Basaia, $\mathrm{PhD}^{1}$; Edoardo G. Spinelli, $\mathrm{MD}^{1,2}$; Elisa Canu, $\mathrm{PhD}^{1}$; Veronica \\ Castelnovo, MSc ${ }^{1,2}$; Nilo Riva, MD, $\mathrm{PhD}^{3}$; Giordano Cecchetti, MD ${ }^{1,2,4}$; Francesca Caso, MD, $\mathrm{PhD}^{4}$; \\ Giuseppe Magnani, $\mathrm{MD}^{4}$; Andrea Falini, $\mathrm{MD}, \mathrm{PhD}^{2,5}$; Massimo Filippi, MD ${ }^{1,2,3,4,6}$; Federica Agosta, \\ $\mathrm{MD}, \mathrm{PhD}^{1,2,4}$
}

This is an open access article distributed under the terms of the Creative Commons Attribution-NonCommercial-NoDerivatives License 4.0 (CC BY-NC-ND), which permits downloading and sharing the work provided it is properly cited. The work cannot be changed in any way or used commercially without permission from the journal.

Neurology® Published Ahead of Print articles have been peer reviewed and accepted for publication. This manuscript will be published in its final form after copyediting, page composition, and review of proofs. Errors that could affect the content may be corrected during these processes. 
Corresponding Author:

Federica Agosta

agosta.federica@hsr.it

Affiliation Information for All Authors: 1. Neuroimaging Research Unit, Division of Neuroscience, IRCCS San Raffaele Scientific Institute, Milan, Italy; 2. Vita-Salute San Raffaele University, Milan, Italy; 3. Neurorehabilitation Unit, IRCCS San Raffaele Scientific Institute, Milan, Italy; 4. Neurology Unit, IRCCS San Raffaele Scientific Institute, Milan, Italy; 5. Neuroradiology Unit and CERMAC, IRCCS San Raffaele Scientific Institute, Milan, Italy; 6. Neurophysiology Service, IRCCS San Raffaele Scientific Institute, Milan, Italy

\section{Contributions:}

Camilla Cividini: Drafting/revision of the manuscript for content, including medical writing for content; Study concept or design; Analysis or interpretation of data; Additional contributions: Statistical analysis Silvia Basaia: Drafting/revision of the manuscript for content, including medical writing for content; Study concept or design; Analysis or interpretation of data Edoardo G. Spinelli: Drafting/revision of the manuscript for content, including medical writing for content; Major role in the acquisition of data; Analysis or interpretation of data

Elisa Canu: Drafting/revision of the manuscript for content, including medical writing for content; Major role in the acquisition of data; Analysis or interpretation of data

Veronica Castelnovo: Drafting/revision of the manuscript for content, including medical writing for content; Major role in the acquisition of data; Analysis or interpretation of data

Nilo Riva: Drafting/revision of the manuscript for content, including medical writing for content; Major role in the acquisition of data; Analysis or interpretation of data

Giordano Cecchetti: Drafting/revision of the manuscript for content, including medical writing for content; Major role in the acquisition of data; Analysis or interpretation of data

Francesca Caso: Drafting/revision of the manuscript for content, including medical writing for content; Major role in the acquisition of data; Analysis or interpretation of data

Giuseppe Magnani: Drafting/revision of the manuscript for content, including medical writing for content; Major role in the acquisition of data; Analysis or interpretation of data

Andrea Falini: Drafting/revision of the manuscript for content, including medical writing for content; Major role in the acquisition of data; Analysis or interpretation of data

Massimo Filippi: Drafting/revision of the manuscript for content, including medical writing for content; Study concept or design; Analysis or interpretation of data; Additional contributions: obtained funding Federica Agosta: Drafting/revision of the manuscript for content, including medical writing for content; Study concept or design; Analysis or interpretation of data; Additional contributions: obtained funding.

Number of characters in title: 122

Abstract Word count: 332

Word count of main text: 4500

References: 50

Figures: 5

Tables: 2

Supplemental: eTables; eReferences; STROBE checklist

Statistical Analysis performed by: Camilla Cividini, MSc, Neuroimaging Research Unit, Division of Neuroscience, IRCCS San Raffaele Scientific Institute, Milan, Italy 
Search Terms: [ 29 ] Frontotemporal dementia, [ 121 ] fMRI, [ 178 ] Amyotrophic lateral sclerosis

Acknowledgements: We thank the patients and their families for the time and effort they dedicated to the research.

Study Funding: The Italian Ministry of Health (GR-2011-02351217; GR-2013-02357415; RF-201102351193); AriSLA (ConnectALS); European Research Council (StG-2016_714388_NeuroTRACK).

Disclosures: The authors C. Cividini, S. Basaia, E.G. Spinelli, E. Canu, V. Castelnovo, N. Riva, G. Cecchetti, F. Caso, G. Magnani, A. Falini report no disclosures relevant to the manuscript; M. Filippi is Editor-in-Chief of the Journal of Neurology, Associate Editor of Human Brain Mapping, Associate Editor of Radiology, and Associate Editor of Neurological Sciences, received compensation for consulting services and/or speaking activities from Alexion, Almirall, Bayer, Biogen, Celgene, Eli Lilly, Genzyme, Merck-Serono, Novartis, Roche, Sanofi, Takeda, and Teva Pharmaceutical Industries, and receives research support from Biogen Idec, Merck-Serono, Novartis, Roche, Teva Pharmaceutical Industries, Italian Ministry of Health, Fondazione Italiana Sclerosi Multipla, and ARiSLA (Fondazione Italiana di Ricerca per la SLA); F. Agosta is Associate Editor of NeuroImage: Clinical, received compensation for consulting services and/or speaking activities from Novartis, Biogen Idec, Philips and Roche, and receives or has received research supports from the Italian Ministry of Health, AriSLA (Fondazione Italiana di Ricerca per la SLA), and the European Research Council. 


\begin{abstract}
Objectives. A significant overlap between amyotrophic lateral sclerosis (ALS) and behavioral variant of frontotemporal dementia (bvFTD) has been observed at clinical, genetic and pathological levels. Within this continuum of presentations, the presence of mild cognitive and/or behavioral symptoms in ALS patients has been consistently reported, although it is unclear whether this is to be considered a distinct phenotype or, rather, a natural evolution of ALS. Here, we used mathematical modeling of MRI connectomic data to decipher common and divergent neural correlates across the ALS-FTD spectrum.
\end{abstract}

Methods. We included 83 ALS patients, 35 bvFTD patients and 61 healthy controls, who underwent clinical, cognitive and MRI assessments. ALS patients were classified according to the revised Strong criteria into 54 ALS with only motor deficits (ALS-cn), 21 ALS with cognitive and/or behavioral involvement (ALS-ci/bi), and 8 ALS with bvFTD (ALS-FTD). First, we assessed the functional and structural connectivity patterns across the ALS-FTD spectrum. Second, we investigated whether and where MRI connectivity alterations of ALS patients with any degree of cognitive impairment (i.e., ALS-ci/bi and ALS-FTD) resembled more the pattern of damage of one (ALS-cn) or the other end (bvFTD) of the spectrum, moving from group-level to single-subject analysis.

Results. As compared with controls, extensive structural and functional disruption of the frontotemporal and parietal networks characterized bvFTD (bvFTD-like pattern), while a more focal structural damage within the sensorimotor-basal ganglia areas characterized ALScn (ALS-cn-like pattern). ALS-ci/bi patients demonstrated an "ALS-cn-like" pattern of structural damage, diverging from ALS-cn with similar motor impairment for the presence of enhanced functional connectivity within sensorimotor areas and decreased functional 
connectivity within the "bvFTD-like" pattern. On the other hand, ALS-FTD patients resembled both structurally and functionally the bvFTD-like pattern of damage with, in addition, the structural ALS-cn-like damage in the motor areas.

Conclusions. Our findings suggest a maladaptive role of functional rearrangements in ALSci/bi concomitantly with similar structural alterations compared to ALS-cn, supporting the hypothesis that ALS-ci/bi might be considered as a phenotypic variant of ALS, rather than a consequence of disease worsening. 


\section{INTRODUCTION}

Amyotrophic lateral sclerosis (ALS) is the most common clinical presentation of motor neuron disease, characterized by progressive neurodegeneration of upper and lower motor neurons. A growing body of evidence supports the notion of clinical, pathological and genetic overlap between ALS and the wide spectrum of frontotemporal dementia (FTD). ${ }^{1}$ Indeed, at least $50 \%$ of ALS patients develop cognitive symptoms - mostly affecting executive functions - and behavioral alterations along the course of the disease, leading to a full-blown diagnosis of FTD in 5-25\% of cases. ${ }^{2,3}$ Considering that comorbid cognitive impairment is a known negative prognostic factor associated with more rapid progression to death or tracheostomy in ALS patients, ${ }^{4,5}$ a better definition and understanding of this condition has clear clinical relevance.

The revised Strong criteria ${ }^{2}$ established a recognized nomenclature for the ALS-FTD clinical continuum ranging from ALS cognitively normal (ALS-cn) to ALS with FTD (ALSFTD), including ALS with cognitive impairment (ALSci), ALS with behavioural impairment (ALSbi), and ALS with combined cognitive and behavioural impairment (ALS-cbi).

Nevertheless, there is currently great debate regarding the pathological underpinnings distinguishing ALS-cn from ALS-ci/bi and ALS-FTD cases, and whether this is to be considered a distinct phenotype or, rather, a natural evolution of ALS. Cross-sectional studies reported an increasing percentage of ALS-ci/bi in disease stages with more severe motor impairment, ${ }^{6}$ and even a sequential cognitive staging system has been proposed for ALS, ${ }^{7}$ mirroring regions involved in pathological stages of TDP-43 deposition. ${ }^{8}$ However, findings of the few available longitudinal neuropsychological studies in ALS diverge, as some support a stability of cognitive and behavioral changes over time, when present, ${ }^{9,10}$ whereas others suggest a subtle progression of cognitive deficits. ${ }^{11,12}$ The largest study in this context ${ }^{5}$ showed that patients who were cognitively impaired at baseline had a faster decline, in 
contrast with a tendency to remain cognitively intact in those who were cognitively unimpaired at study entry.

In this context, advanced magnetic resonance imaging (MRI) has provided a useful tool to investigate brain architecture in ALS and FTD. Several MRI studies evaluated patients with behavioral variant of FTD (bvFTD), using both conventional MRI ${ }^{13-16}$ and connectomic approaches, ${ }^{17,18}$ reporting specific patterns of structural and functional damage within frontoinsular and temporal networks. In ALS, widespread grey matter (GM) ${ }^{19-21}$ and white matter (WM) damage ${ }^{19,20,22}$ has been shown in patients cognitively impaired relative to ALScn patients, involving not only motor but also extra-motor areas, including frontotemporal, parietal, insular and cingulate regions. A recent study using a connectomic approach revealed widespread cerebral WM changes affecting frontotemporal regions in ALS-ci/bi patients relative to ALS-cn patients. ${ }^{23}$ Available functional MRI studies have reported conflicting results, as executive dysfunction and behavioral disturbances in ALS have been associated with either enhanced functional connectivity in frontoparietal and temporal networks ${ }^{24-26}$ or suppressed connectivity within frontoparietal, salience and executive networks. ${ }^{15,27}$ However, in the current literature, there is a lack of MRI studies specifically assessing functional brain alterations in ALS with mild cognitive/behavioral decline, as only one study suggested an enhanced functional connectivity in patients with cognitive decline relative to ALS-cn. ${ }^{28}$ To date, a direct evaluation of brain network reorganization in ALS-ci/bi compared with the opposite ends of the ALS-FTD spectrum (i.e., ALS-cn and full-blown FTD) is still needed. Moreover, no studies have combined the structural and functional information using graph analysis and connectomics to investigate neural correlates of cognitive and behavioral decline within patients of the spectrum. The aim of the present study was to bridge this gap, investigating structural and functional network correlates of cognitive/behavioral impairment in patients within the ALS-FTD continuum, who were fully characterized according to the revised Strong criteria. ${ }^{2}$ Using up-to-date MRI approaches, we assessed distinctive patterns of 
network disruption (i.e., "ALS-cn-like pattern" and "bvFTD-like pattern") that may prove useful for accurate classification at a single-patient level.

\section{METHODS}

An overview of the Methods is provided in Figure 1.

\section{Participants}

Eighty-three ALS and 35 bvFTD patients were recruited at the IRCCS Ospedale San Raffaele, Milan, Italy, in the framework of an observational study (Figure 1.I). Only sporadic patients (i.e., with no family history of dementia or motor neuron disease) who proved negative for mutations in the major genes associated with ALS/FTD (i.e., C9ORF72, GRN, MAPT, TARDBP, SOD1, FUS, TBK1, TREM2, OPTN and $V C P)$ were included. The diagnosis of ALS was based on the revised El Escorial criteria, ${ }^{29}$ whereas bvFTD was diagnosed according to Rascovsky criteria. ${ }^{30}$ Patients underwent a comprehensive evaluation including neurological history, clinical assessment (Table 1), neuropsychological testing (eTable 1, http://links.lww.com/WNL/B674) and MRI scan. For ALS patients, the site of disease onset was recorded; disease severity was assessed using the ALSFRS-r; ${ }^{31}$ rate of disease progression was defined as [48-ALSFRS-r score]/time from symptom onset; and muscular strength was assessed by manual muscle testing based on the Medical Research Council (MRC) scale. ALS patients were receiving riluzole at study entry. For bvFTD

patients, disease severity was assessed using the Clinical Dementia Rating scale. ${ }^{32}$

Sixty-one healthy controls were recruited by word of mouth, based on the following criteria: no history of neurologic and psychiatric diseases, no family history of neurodegenerative diseases, and a normal neurological assessment (Table 1).

Exclusion criteria for all subjects were: (other) significant medical illnesses or substance abuse that could interfere with cognitive functioning; any (other) major systemic, psychiatric, or neurological illnesses; and other causes of focal or diffuse brain damage, including lacunae and extensive cerebrovascular disorders at routine MRI. 


\section{Cognitive and Behavioral Assessment}

Patient classification (Figure 1.I). Comprehensive multi-domain cognitive testing was performed by trained neuropsychologists unaware of MRI results. Tested cognitive domains were: global cognitive functioning, memory, executive function, visuospatial abilities, fluency, language, mood and behaviors, as previously described ${ }^{18,25}$ (eTable 1 , http://links.lww.com/WNL/B674). According to the revised Strong criteria, ${ }^{2}$ patients with ALS were classified into 54 cases with motor impairment only (ALS-cn), 21 cases with cognitive and/or behavioral deficits (ALS-ci/bi) and 8 ALS patients with bvFTD (ALS-FTD).

\section{MRI acquisition and pre-processing}

MRI scans were obtained using a 3T Philips Medical Systems Intera machine scan. T1-weighted, T2-weighted, fluid-attenuated inversion recovery, diffusion tensor MRI (DT MRI) and resting-state functional MRI (RS fMRI) sequences were acquired. Full details of the MRI acquisition protocol are reported in eTable 2, http://links.lww.com/WNL/B674. MRI analyses were performed by experienced observers blinded to subjects' identity.

Connectome Reconstruction (Figure 1.II). Brain parcellation, DT MRI and RS fMRI pre-processing, and construction of brain structural and functional connectome have been

described previously. ${ }^{18,25}$ Briefly, brain was parcellated into 220 similarly-sized GM cortical and subcortical regions (eTable 3, http://links.lww.com/WNL/B674). Applying a graph theoretical approach, the 220 brain regions are represented as nodes and structural/functional connections linking each pair of nodes as edges. Edges for structural connectivity are represented by fractional anisotropy (FA), whereas functional edges are represented by Pearson's correlation coefficients between each pair of nodes. Once the structural macroscale connectome was reconstructed per each subject, we applied the structural connectome of an independent healthy control group as a comprehensive brain connection mask. ${ }^{18}$ Then, the 
masked structural connectome of each subject was used as mask for the respective functional connectome, in order to investigate the functional alterations only where structural connections exist, enhancing the biological interpretation of the results. ${ }^{33}$

\section{Statistical analysis}

\section{Characterization of functional and structural connectivity across the ALS-FTD spectrum}

Regional connectivity analysis (Figure 1.III). We investigated structural and

functional network features in the different subject groups at regional level. Network Based Statistic (NBS) ${ }^{34}$ was performed to assess regional structural and functional connectivity strength at the level of significance $\mathrm{p}<0.05$. All possible combinations of comparisons between groups were performed. The largest (or principal) connected component and the smaller clusters of altered connections were identified. ${ }^{25,34}$ A corrected p-value was calculated for each contrast using an age-, sex-, and education-adjusted permutation analysis (10000 permutations).

\section{Investigation of ALS-cn-like or bvFTD-like patterns of alterations in ALS-ci/bi and ALS-} FTD

The following analyses were focused firstly on identifying the specific structural and functional connectivity patterns that characterize the ends of the ALS-FTD spectrum (ALScn and bvFTD). Secondly, we investigated whether and where ALS-ci/bi and ALS-FTD patients showed an ALS-cn-like or a bvFTD-like connectivity pattern.

Distribution analysis (Figure 1.IV). Distribution analysis was performed to assess the structural and functional connectivity alterations in patient groups. The connectivity values of each connection for each patient were normalized relative to controls as follows:

$$
Z_{i j}^{S}=\frac{C_{i j}^{S}-\mu}{\sigma}
$$


where $C_{i j}^{S}$ is the structural/functional connectivity value of the connection between node $i$ and $j$ for subject $s ; \mu$ is the mean structural/functional connectivity value of the considered connection in the control group; and $\sigma$ is the standard deviation of the structural/functional connectivity value of such connection in the control group. Subsequently, the 220 regions from both hemispheres were grouped into six anatomical macro-areas (hereafter referred to as brain areas): temporal, parietal, occipital, fronto-insular, basal ganglia, and sensorimotor. Per each patient group (ALS-cn, ALS-ci/bi, ALS-FTD, and bvFTD), the mean values of intra- and inter-area connectivity were calculated averaging the normalized structural/functional connections belonging to an area (intra) or linking two distinct areas (inter), respectively. The percentage of patients with connectivity value below the reference value (i.e., control mean value) was calculated per each intra- and inter-area network. Finally, the intra- and inter-area connectivity values were compared between patient groups using age-, sex-, and education-adjusted analysis of variance models, followed by post hoc pairwise comparisons, Bonferroni-corrected for multiple comparisons $(\mathrm{p}<0.05$, SPSS Statistics 26.0 [SPSS Inc., Chicago, IL]).

Classification analysis (Figure 1.V). Classification analysis was performed to define the characteristic structural/functional patterns of damage of the two ends of the spectrum (ALS-cn and bvFTD). For this purpose, we selected the structural and functional connectivity values only in those intra- and inter-area networks, where ALS-cn and bvFTD showed significantly different patterns in the distribution analysis. Receiver Operator Characteristic (ROC) curve analysis was performed in these selected networks. The area under the curve (AUC), as derived measure of accuracy, was considered to assign a specific set of structural/functional alterations to ALS-cn (ALS-cn-like pattern) or to bvFTD (bvFTD-like pattern). Per each intra- and/or inter-area connectivity value involved in one of the two patterns, Youden Index was calculated, providing the best tradeoff between sensitivity and 
specificity. Finally, patients of each group were classified in those with connectivity values above or below the identified optimal cut-offs.

Frequency analysis (Figure 1.VI). Aiming to assess, at the single-subject level, whether and where ALS-ci/bi and ALS-FTD patients showed commonalities and differences with ALS-cn-like or bvFTD-like patterns, we performed a frequency analysis using the Chisquared test $(\mathrm{p}<0.05)$. Specifically, we identified and compared between groups the frequency of subjects with connectivity values above and below the optimal cut-offs belonging to the ALS-cn-like and the bvFTD-like pattern. ALS-cn group was excluded in the frequency analysis of the ALS-cn-like pattern, as well as the bvFTD group was not considered in the bvFTD-like pattern analysis.

\section{Data availability}

The dataset used during the current study will be made available by the corresponding author upon request to qualified researchers (i.e., affiliated to a university or research institution/hospital).

\section{Standard Protocol Approvals, Registration, and Patient Consents}

Local ethical standards committee on human experimentation approved the study protocol and all participants (or their caregivers) provided written informed consent.

\section{RESULTS}

\section{Clinical and neuropsychological features}

Demographic and clinical characteristics of study groups are reported in Table 1, while neuropsychological features in eTable 1, http://links.lww.com/WNL/B674. Relative to controls, ALS-cn and bvFTD patients showed a larger proportion of male individuals. In addition, ALS-ci/bi and bvFTD patients showed lower education relative to controls. ALS groups and bvFTD patients were different for disease duration at MRI, which was shorter in 
ALS patients. ALS groups were comparable in terms of disease severity, as assessed by ALSFRS-r and MRC global score, disease progression rate and site of clinical onset, although ALS-ci/bi were older than ALS-cn. The neuropsychological assessment did not reveal differences between controls and ALS-cn. bvFTD and ALS-FTD patients performed worse than controls and ALS-cn cases in all investigated cognitive domains. The ALS-ci/bi group performed worse than controls in naming (actions) and better than bvFTD and ALSFTD patients in fluency tests, with additional higher performance in global cognition, verbal memory, and abstract reasoning compared to bvFTD group only (eTable 1, http://links.lww.com/WNL/B674).

\section{Characterization of functional and structural connectivity across the ALS-FTD} spectrum (Figure 2)

Structural connectivity. Regional connectivity analysis showed alterations involving the connections within and among the sensorimotor network, basal ganglia, frontal, temporal and parietal areas, in addition to minimal involvement of the occipital connections, in ALScn patients relative to controls $(\mathrm{p}=0.01$; Figure $2 \mathrm{~A}[1])$. This structural pattern of damage was also found in ALS-ci/bi and ALS-FTD cases relative to controls ( $\mathrm{p}=0.02$ and $\mathrm{p}=0.001$, Figure $2 \mathrm{~A}[2,3]$, respectively), with a more widespread disruption of the same networks in ALS-FTD reflecting increasing severity of impaired behavior and cognition (Figure 2A[3]). ALS-FTD patients showed also a more severe structural damage, mainly within frontal areas, relative to ALS-cn cases ( $\mathrm{p}=0.01$; Figure 2A[6]). Additionally, ALS-cn patients showed greater structural alterations relative to bvFTD ( $\mathrm{p}=0.03$; Figure $2 \mathrm{~A}[5])$ in few connections within and among sensorimotor regions, parietal areas, and basal ganglia, especially involving thalamus and those connections from pallidum and putamen towards precentral, postcentral and precuneus bilaterally. Patients with bvFTD showed a widespread structural damage relative 
to controls, ALS-cn and ALS-ci/bi patients across the whole brain $(\mathrm{p}<0.001$; Figure $2 \mathrm{~A}[4,7,8]$, respectively). No further differences were observed in the remaining comparisons.

Functional connectivity. NBS analysis did not show differences in functional connectivity in ALS groups relative to controls, although ALS-ci/bi patients showed a trend toward an enhanced functional connectivity relative to controls within frontal and basal ganglia areas $(\mathrm{p}=0.06)$. On the other hand, bvFTD patients were characterized by reduced functional connectivity relative to controls $(\mathrm{p}=0.02$; Figure $2 \mathrm{~B}[1])$, ALS-cn $(\mathrm{p}=0.01$; Figure 2B[3)) and ALS-ci/bi ( $<<0.001$; Figure 2B[4]) cases, mainly involving the connections within the frontotemporal regions and between frontal and sensorimotor areas. ALS-FTD relative to ALS-ci/bi patients showed reduced functional connectivity within and between the frontal, temporal and motor areas similarly to bvFTD cases $(p=0.02$; Figure $2 B[2])$. No further differences were observed in the remaining comparisons.

\section{Investigation of ALS-cn-like and bvFTD-like patterns of alterations in ALS-ci/bi and}

\section{ALS-FTD: structural connectivity (Figure 3-4)}

Distribution analysis. Compared with ALS-cn, bvFTD patients showed greater structural intra-area disruption within frontal, temporal and parietal areas (Figure 3[1], Figure 4[3,4] and eTable 4, http://links.lww.com/WNL/B674; p<0.05) and inter-area disruption in the frontal, temporal and occipital connections toward parietal lobe $(\mathrm{p}=0.01$, Figure $3[2]$ and Figure $4[2,5]$ ), in the frontal, basal ganglia and occipital connections toward temporal areas $(p=0.002, p<0.001$ and $p=0.03$, Figure 3[3] and Figure 4[1,6] respectively), and in the connections between frontal and basal ganglia $(\mathrm{p}<0.001)$ (Figure 3[5] and eTable 4, http://links.lww.com/WNL/B674). Most of bvFTD patients (from 83 to 100\%) were found severely disrupted in these networks (eTable 4, http://links.lww.com/WNL/B674). On the other hand, most of ALS-cn patients (81\%) were characterized by a greater damage within the motor network, specifically among the sensorimotor - basal ganglia connections, relative 
to bvFTD cases ( $\mathrm{p}=0.01$, Figure 3[7]). Additionally, ALS-FTD patients showed structural connectivity alterations within the motor areas, resembling the ALS-cn damage. In particular, $88 \%$ of ALS-FTD revealed a significant structural disruption in the sensorimotor-basal ganglia connections compared with bvFTD ( $\mathrm{p}=0.01$; Figure 3[7] and eTable 4, http://links.lww.com/WNL/B674). Among the other brain regions, ALS-ci/bi and ALS-FTD patients behaved differently. ALS-ci/bi patients showed significant structural connectivity differences within frontal and temporal lobe (Figure 3[1] and Figure 4[3]) and between frontal, temporal and basal ganglia areas compared to bvFTD $(p<0.05$, (Figure $3[3,5]$ and Figure 4[1]), embracing a pattern of damage more like ALS-cn. On the other hand, ALS-FTD revealed a behavior more like bvFTD, showing a greater structural disruption within frontal $(\mathrm{p}=0.03)$ and in frontal - sensorimotor connections $(\mathrm{p}=0.02)$ compared to ALS-cn (Figure 3[1,4] and eTable 4, http://links.lww.com/WNL/B674).

Classification analysis. From ROC curve analysis, two characteristic patterns of damage were identified: the "ALS-cn-like pattern" defined by a focal structural damage within sensorimotor-basal ganglia areas that distinguished ALS-cn from bvFTD patients (accuracy $[\mathrm{AUC}]=0.67$, eFigure. 1A-blue line), and the "bvFTD-like pattern" characterized by structural alterations of the frontotemporal and parietal networks that discriminated bvFTD from ALS-cn cases with AUC ranging from 0.67 and 0.88 (eFigure. 1A-red lines, http://links.lww.com/WNL/B674). The best cutoff of structural connectivity per each significant network are reported in Table 2.

Frequency analysis. The ALS-cn-like pattern was identified more frequently in ALSci/bi and ALS-FTD compared with bvFTD patients (ALS-ci/bi $v s$ bvFTD p=0.04; ALS-FTD vs bvFTD non-significant trend $\mathrm{p}=0.07)$ (eTable 5, http://links.lww.com/WNL/B674). On the other hand, the bvFTD-like pattern was found to be more frequent neither in ALS-ci/bi nor ALS-FTD compared to ALS-cn, except for a non-significant trend $(\mathrm{p}=0.08)$ within frontal 
and among frontal-basal ganglia, temporal-occipital areas in ALS-FTD relative to ALS-cn cases (eTable 5, http://links.lww.com/WNL/B674).

\section{Investigation of ALS-cn-like and bvFTD-like patterns of alterations in ALS-ci/bi and}

\section{ALS-FTD: functional connectivity (Figure 5)}

Distribution analysis. Regarding functional connectivity distribution analysis, decreased functional connectivity within frontotemporal $(\mathrm{p}=0.001)$ and between sensorimotor and parietal connections $(\mathrm{p}<0.02)$ was found in bvFTD compared with ALS-cn patients (Figure 5[1,2] and eTable 4, http://links.lww.com/WNL/B674). ALS-ci/bi patients showed significant enhanced functional connectivity relative to bvFTD in the frontal-sensorimotor connections ( $\mathrm{p}=0.001)$, parietotemporal connections $(\mathrm{p}=0.03)$ and within sensorimotor areas ( $p<0.001$, Figure 5[3,4,5] and eTable 4, http://links.lww.com/WNL/B674). Additionally, ALS-ci/bi showed increased functional connectivity within sensorimotor areas relative to ALS-cn ( $p<0.04$, Figure 5[4] and eTable 4). Of note, most ALS-ci/bi patients (a percentage ranging from 67 to $76 \%$ ) revealed normalized values of functional connectivity greater than zero in these abovementioned networks (i.e., frontal-sensorimotor, parietotemporal and sensorimotor). Moreover, ALS-FTD patients showed a significant greater reduced functional connectivity in temporal-sensorimotor connections compared to ALS-cn ( $\mathrm{p}=0.03)$ and ALSci/bi $(\mathrm{p}<0.01$, Figure 5[6] and eTable 4).

Classification analysis. The ROC curve analysis on functional connectivity data identified only a "bvFTD-like pattern" of functional damage, involving frontotemporal and sensorimotor-parietal connections, with an AUC of 0.77 and 0.67 in discriminating bvFTD from ALS-cn, respectively (eFigure. 1B-red lines, http://links.lww.com/WNL/B674). The best cutoff values of functional connectivity for each significant network are reported in Table 2. 
Frequency analysis. Within frontotemporal connections, ALS-ci/bi patients were characterized by a greater proportion of cases showing bvFTD-like decreased functional connectivity compared with ALS-cn ( $\mathrm{p}=0.03$; eTable 5, http://links.lww.com/WNL/B674), but a lower proportion compared with ALS-FTD ( $\mathrm{p}=0.02)$, who mostly showed a typical bvFTD-like pattern with a decreased functional connectivity relative to ALS-cn patients (p<0.001; eTable 5, http://links.lww.com/WNL/B674).

\section{DISCUSSION}

The present multiparametric MRI study provides a comprehensive characterization of the neural correlates across the spectrum of ALS-FTD clinical presentations. A connectomebased approach was adopted, first, to identify the connectivity signatures of ALS-cn and bvFTD (i.e., the two ends of this spectrum) and, subsequently, to characterize the alterations underlying mild cognitive/behavioral deficits and full-blown dementia in ALS patients, with the aid of mathematical models and single-subject analysis. An ALS-cn-like pattern was defined by a focused structural damage within the motor areas. By contrast, a bvFTD-like pattern was delineated by a widespread structural damage and decreased functional connectivity, specifically in frontal, temporal and parietal areas. ALS-ci/bi patients showed a pattern of structural damage mostly overlapping with the ALS-cn-like pattern, whereas functional data diverged from ALS-cn for the presence of enhanced functional connectivity within the sensorimotor regions and decreased functional connectivity in the frontotemporal areas (i.e., mirroring a bvFTD-like pattern). Finally, ALS-FTD resembled the bvFTD-like pattern of damage both structurally and functionally, with, in addition, the structural ALS-cnlike damage in the motor areas. Although connectivity data alone cannot fully address the homogeneity or heterogeneity of this spectrum, our findings suggest a maladaptive role of functional rearrangements in ALS-ci/bi concomitantly with similar structural alterations 
compared to ALS-cn, supporting the hypothesis that ALS-ci/bi might be considered as a phenotypic variant of ALS, rather than a consequence of disease worsening.

When considering the results of the present study, some limitations should be noted. Despite the robust size of the overall ALS cohort, some subgroups were small (i.e., ALSFTD), although this is indicative of the relative incidence of cognitive alterations. This aspect has also influenced our choice to bring together patients with mild cognitive dysfunction (i.e., ALS-ci) and patients with mild behavioral disturbances (i.e., ALS-bi), to avoid dispersion of data and the reduced statistical power that would result. Furthermore, the lack of information of a definite pathological diagnosis for bvFTD patients is an important limitation of the present study, even though the aim of the work was to explore the neural correlates of the clinical rather than the pathological heterogeneity of the ALS-FTD spectrum. Another issue lies in the cross-sectional nature of the study. In this context, longitudinal studies are warranted to verify whether cognitive/behavioral dysfunction is a stable or progressive feature of the ALS trajectory, and to assess the evolution of associated network alterations over time.

The inherent limitations of MRI connectomic should also be acknowledged ${ }^{35,36}$ including, among others, the lack of an optimal framework, i.e., a reference standard for the regional parcellation of brain MR imaging. It is also important to note that the accuracy of any attempt to model the connectome is biased by the intrinsic limitations of the imaging techniques used. For example, fibre tracking based on DT MRI is known to be poor at points where only limited information about the WM fibre direction is available such as where multiple tracts cross. This results in incomplete reconstruction of tracts and a general underrepresentation of long-distance connections in the brain. Despite these shortcomings, our study highlights the potential of multiparametric connectome-based approaches for providing novel pathophysiological insights and biomarkers of cognitive dysfunction in the context of ALS-FTD. A key point of our study was the demonstration of characteristic brain structural 
damage and functional rearrangements across ALS cognitive phenotypes, as defined based on the application of revised Strong criteria to a sizeable monocentric cohort. Our conclusions were made possible by the extensive clinical and neuropsychological characterization of the sample, as well as by the multiparametric nature of this study. Current MRI literature has generally provided results based on the assessment of structural and functional alterations separately, at voxel or regional level, without a straightforward investigation of their relationship. Conversely, a connectomic approach gave us the potential to bridge the gap of the anatomo-functional link thanks to the application of the same parcellation system, connectome reconstruction framework and statistical approach. Whereas the capability of connectome-based approach to provide information on the brain network architecture was achieved by a group-level analysis, smoothing out the inter-individual variability, a further innovative aspect of our study was the transition to the single-level analysis by the help of mathematical models. Indeed, the study framework was able to identify the ALS-cn-like or bvFTD-like patterns of damage, and to characterize the type of damage that each ALS-ci/bi and ALS-FTD patient shared with such signatures of network alterations.

The selective involvement of motor WM regions in the ALS-cn sample is largely consistent with previous literature, ${ }^{21,25,37}$ confirming a "signature" pattern of frank decline in FA of the subnetworks connecting primary motor, supplementary motor and premotor areas, as well as basal ganglia - specifically, the thalamus. ${ }^{38}$ The structural disruption of the sensorimotor network supports the current view of this network as the epicenter of degenerative process of the disease, in line with proposed neuropathological and MRI-based disease staging systems. ${ }^{8,39}$ As for the functional MRI findings, the current literature counts on a number of studies reporting reduced ${ }^{15,27}$ or increased functional connectivity in ALS patients, ${ }^{24-26}$ or even a mixed picture. ${ }^{40}$ Nevertheless, there is a shortage of MRI studies focusing on functional brain rearrangements in ALS related to cognitive status, and our findings contribute to fill this gap. Of note, both regional (i.e., NBS) and distribution analyses 
suggest that ALS-cn patients are characterized by a quite preserved functional connectivity comparable to the functional healthy-brain organization.

The bvFTD-like pattern included a widespread brain structural disruption, with a predominant damage in the frontotemporoparietal network and the involvement of the striatum, and functional connectivity breakdown within the same networks. Our findings confirm previous evidence that see the disconnection of the frontoinsular and temporal regions as hallmark of the behavioral clinical syndrome of FTD both at structural and functional levels. ${ }^{14,16-18}$ Herein, we extend these results by highlighting the relative preservation of the motor areas in bvFTD, in contrast with a widespread structural and functional involvement of the anterior frontal lobes, as well as a differential involvement of the basal ganglia circuits when compared with ALS-cn (i.e., greater involvement of striatal connections in bvFTD, in contrast with thalamic involvement in ALS-cn). These findings are in line with previous reports, ${ }^{38,41}$ and support the notion of a diverging network vulnerability to disease pathology in the two opposite ends of the ALS-FTD spectrum.

The focus of the current study was on elucidating MRI connectomic underpinnings of mild or full-blown cognitive deficits in ALS, possibly addressing the long-standing debate on the nature of cognitive deficits in the course of the disease, as an early or, rather, a late-stage feature. Regarding the structural brain network, the presence of mild cognitive and/or behavioral impairment in ALS patients did not contribute significantly to an additional microstructural damage relative to ALS-cn with otherwise comparable clinical characteristics - including measures of motor impairment and disease duration. Although previous literature has suggested greater structural damage related to cognitive impairment in ALS, ${ }^{19,20,21-23}$ such damage was generally subtle and possibly driven by the inclusion of ALS-FTD subjects. By contrast, our study highlighted shared structural damage between ALS-ci/bi and ALS-cn patients, involving mainly the motor networks. On the other hand, the analysis of functional connectivity alterations played an important role for the differentiation of ALS-ci/bi from 
ALS-cn. Indeed, ALS-ci/bi patients showed a rearrangement of the functional networks, which was divergent from ALS-cn, with enhanced functional connectivity within motor areas and decreased connectivity in the frontotemporal networks. The concomitant absence of significant structural alterations, compared with the ALS-cn group, apparently supports a maladaptive role of such functional rearrangements in ALS-ci/bi, as previously hypothesized..$^{25,42}$ The biological underpinnings of such functional disequilibrium have been suggested to lie in the known excitatory/inhibitory imbalance due to interneuron pathology in ALS, causing a reduction in recurring inhibition that has been associated with disease severity. ${ }^{43,44}$ We argue that functional imbalance between motor and extra-motor frontal networks might be particularly severe in ALS-ci/bi, causing mild cognitive disturbances even in early phases of the disease - consistent with the relatively short disease duration of the present cohort. Therefore, our data suggest that ALS-ci/bi might be considered as a phenotypic variant of ALS, rather than a consequence of disease worsening. ${ }^{6,7}$ These findings may find support in one of the few longitudinal neuropsychological studies in this context, ${ }^{5}$ in which cognition decline was faster in patients who were already cognitively impaired at baseline, while normal cognition tended to remain intact with slower motor and cognitive progression. Of note, education levels of ALS-ci/bi patients were lower than ALS-cn in our sample, consistent with the recently highlighted influence of environmental factors that collectively constitute the cognitive reserve (i.e., education, occupation and physical activity) over an early development of cognitive symptoms in ALS. ${ }^{45}$

In contrast with ALS-ci/bi cases, when ALS patients had co-occurrent dementia (ALS-FTD), our study has outlined not only a pattern of microstructural damage involving the motor networks (i.e., the characteristic ALS-cn-like pattern), but also a disruption of frontal, temporal, parietal and striatal circuits, both from a structural and a functional point of view - therefore, resembling the bvFTD-like pattern. ${ }^{46}$ These findings agree with the pattern of widespread hypometabolism recently demonstrated in ALS cases with severe cognitive 
impairment, ${ }^{47}$ possibly mirroring the most advanced stages of TDP-43 neuropathological models which have been proposed both in $\mathrm{ALS}^{8}$ and bvFTD, ${ }^{48}$ here sharing the same pathological signature. ${ }^{49,50}$ Similar to ALS-ci/bi, ALS-FTD patients showed similar severity of motor symptoms and disease duration when compared with ALS-cn, supporting a view of this clinical presentation as a specific phenotype within the frontotemporal lobar degeneration spectrum, characterized by a combined, severe involvement of both motor and extra-motor brain networks, rather than an evolution of either ALS or bvFTD. 
Appendix 1. Authors

\begin{tabular}{|c|c|c|}
\hline Name & Location & Contribution \\
\hline $\begin{array}{l}\text { Camilla } \\
\text { Cividini, MSc }\end{array}$ & $\begin{array}{l}\text { Neuroimaging Research } \\
\text { Unit, Division of } \\
\text { Neuroscience, IRCCS San } \\
\text { Raffaele Scientific Institute, } \\
\text { and Vita-Salute San } \\
\text { Raffaele University, Milan, } \\
\text { Italy }\end{array}$ & $\begin{array}{l}\text { Design and } \\
\text { conceptualization of study; } \\
\text { analysis and interpretation of } \\
\text { data; drafted and revised the } \\
\text { manuscript for intellectual } \\
\text { content. }\end{array}$ \\
\hline $\begin{array}{l}\text { Silvia Basaia, } \\
\text { PhD }\end{array}$ & $\begin{array}{l}\text { Neuroimaging Research } \\
\text { Unit, Division of } \\
\text { Neuroscience, IRCCS San } \\
\text { Raffaele Scientific Institute }\end{array}$ & $\begin{array}{l}\text { Design and } \\
\text { conceptualization of study; } \\
\text { analysis and interpretation of } \\
\text { data; drafted and revised the } \\
\text { manuscript for intellectual } \\
\text { content. }\end{array}$ \\
\hline $\begin{array}{l}\text { Edoardo G } \\
\text { Spinelli, MD }\end{array}$ & $\begin{array}{l}\text { Neuroimaging Research } \\
\text { Unit, Division of } \\
\text { Neuroscience, IRCCS San } \\
\text { Raffaele Scientific Institute, } \\
\text { and Vita-Salute San } \\
\text { Raffaele University, Milan, } \\
\text { Italy }\end{array}$ & $\begin{array}{l}\text { Acquisition and } \\
\text { interpretation of data; } \\
\text { drafted and revised the } \\
\text { manuscript for intellectual } \\
\text { content. }\end{array}$ \\
\hline $\begin{array}{l}\text { Elisa Canu, } \\
\text { PhD }\end{array}$ & $\begin{array}{l}\text { Neuroimaging Research } \\
\text { Unit, Division of } \\
\text { Neuroscience, IRCCS San } \\
\text { Raffaele Scientific Institute }\end{array}$ & $\begin{array}{l}\text { Acquisition and } \\
\text { interpretation of data; } \\
\text { revised the manuscript for } \\
\text { intellectual content. }\end{array}$ \\
\hline $\begin{array}{l}\text { Veronica } \\
\text { Castelnovo, } \\
\text { MSc }\end{array}$ & $\begin{array}{l}\text { Neuroimaging Research } \\
\text { Unit, Division of } \\
\text { Neuroscience, IRCCS San } \\
\text { Raffaele Scientific Institute, }\end{array}$ & $\begin{array}{l}\text { Acquisition and } \\
\text { interpretation of data; } \\
\text { revised the manuscript for } \\
\text { intellectual content. }\end{array}$ \\
\hline
\end{tabular}




\begin{tabular}{|c|c|c|}
\hline & $\begin{array}{l}\text { and Vita-Salute San } \\
\text { Raffaele University, Milan, } \\
\text { Italy }\end{array}$ & \\
\hline $\begin{array}{l}\text { Nilo Riva, MD, } \\
\text { PhD }\end{array}$ & $\begin{array}{l}\text { Neurorehabilitation Unit, } \\
\text { IRCCS San Raffaele } \\
\text { Scientific Institute, Milan, } \\
\text { Italy }\end{array}$ & $\begin{array}{l}\text { Acquisition and } \\
\text { interpretation of data; } \\
\text { revised the manuscript for } \\
\text { intellectual content. }\end{array}$ \\
\hline $\begin{array}{l}\text { Giordano } \\
\text { Cecchetti, MD }\end{array}$ & $\begin{array}{l}\text { Neuroimaging Research } \\
\text { Unit, Division of } \\
\text { Neuroscience, Neurology } \\
\text { Unit, IRCCS San Raffaele } \\
\text { Scientific Institute, and } \\
\text { Vita-Salute San Raffaele } \\
\text { University, Milan, Italy }\end{array}$ & $\begin{array}{l}\text { Acquisition and } \\
\text { interpretation of data; } \\
\text { revised the manuscript for } \\
\text { intellectual content. }\end{array}$ \\
\hline $\begin{array}{l}\text { Francesca } \\
\text { Caso, MD, PhD }\end{array}$ & $\begin{array}{l}\text { Neurology Unit, IRCCS San } \\
\text { Raffaele Scientific Institute }\end{array}$ & $\begin{array}{l}\text { Acquisition and } \\
\text { interpretation of data; } \\
\text { revised the manuscript for } \\
\text { intellectual content. }\end{array}$ \\
\hline $\begin{array}{l}\text { Giuseppe } \\
\text { Magnani, MD }\end{array}$ & $\begin{array}{l}\text { Neurology Unit, IRCCS San } \\
\text { Raffaele Scientific Institute }\end{array}$ & $\begin{array}{l}\text { Acquisition and } \\
\text { interpretation of data; } \\
\text { revised the manuscript for } \\
\text { intellectual content. }\end{array}$ \\
\hline $\begin{array}{l}\text { Andrea Falini, } \\
\text { MD }\end{array}$ & $\begin{array}{l}\text { Department of } \\
\text { Neuroradiology and } \\
\text { CERMAC, Division of } \\
\text { Neuroscience, IRCCS San } \\
\text { Raffaele Scientific Institute, } \\
\text { Milan, Italy }\end{array}$ & $\begin{array}{l}\text { Acquisition and } \\
\text { interpretation of data; } \\
\text { revised the manuscript for } \\
\text { intellectual content. }\end{array}$ \\
\hline $\begin{array}{l}\text { Massimo } \\
\text { Filippi, MD }\end{array}$ & $\begin{array}{l}\text { Neuroimaging Research } \\
\text { Unit, Division of }\end{array}$ & $\begin{array}{l}\text { Design and } \\
\text { conceptualization of study; }\end{array}$ \\
\hline
\end{tabular}




\begin{tabular}{|l|l|l|}
\hline \multirow{7}{*}{$\begin{array}{l}\text { Neuroscience, Neurology } \\
\text { Unit, Neurophysiology } \\
\text { Unit, IRCCS San Raffaele } \\
\text { Scientific Institute, and } \\
\text { Vita-Salute San Raffaele } \\
\text { Aniversity, Milan, Italy }\end{array}$} & $\begin{array}{l}\text { interpretation of data; } \\
\text { drafted and revised the } \\
\text { manuscript for intellectual } \\
\text { content; obtained funding. }\end{array}$ \\
\hline PhD & $\begin{array}{l}\text { Neuroimaging Research } \\
\text { Unit, Division of } \\
\text { Neuroscience, Neurology } \\
\text { Unit, IRCCS San Raffaele } \\
\text { Scientific Institute, and } \\
\text { Vita-Salute San Raffaele } \\
\text { University, Milan, Italy }\end{array}$ & $\begin{array}{l}\text { Design and } \\
\text { conceptualization of study; } \\
\text { interpretation of data; } \\
\text { drand revised the } \\
\text { manuscript for intellectual } \\
\text { content; obtained funding. }\end{array}$ \\
\hline
\end{tabular}




\section{Supplement-http://links.lww.com/WNL/B674}

\section{REFERENCES}

1. Burrell JR, Halliday GM, Kril JJ, et al. The frontotemporal dementia-motor neuron disease continuum. Lancet 2016;388:919-931.

2. Strong MJ, Abrahams S, Goldstein LH, et al. Amyotrophic lateral sclerosis frontotemporal spectrum disorder (ALS-FTSD): Revised diagnostic criteria. Amyotroph Lateral Scler Frontotemporal Degener 2017;18:153-174.

3. Saxon JA, Thompson JC, Jones M, et al. Examining the language and behavioural profile in FTD and ALS-FTD. J Neurol Neurosurg Psychiatry 2017;88:675-680.

4. Calvo A, Moglia C, Lunetta C, et al. Factors predicting survival in ALS: a multicenter Italian study. J Neurol 2017;264:54-63.

5. Elamin M, Bede P, Byrne S, et al. Cognitive changes predict functional decline in ALS: a population-based longitudinal study. Neurology 2013;80:1590-1597.

6. Chio A, Moglia C, Canosa A, et al. Cognitive impairment across ALS clinical stages in a population-based cohort. Neurology 2019;93:e984-e994.

7. Lule D, Bohm S, Muller HP, et al. Cognitive phenotypes of sequential staging in amyotrophic lateral sclerosis. Cortex 2018;101:163-171.

8. Brettschneider J, Del Tredici K, Toledo JB, et al. Stages of pTDP-43 pathology in amyotrophic lateral sclerosis. Ann Neurol 2013;74:20-38.

9. Kasper E, Schuster C, Machts J, et al. Dysexecutive functioning in ALS patients and its clinical implications. Amyotroph Lateral Scler Frontotemporal Degener 2015;16:160-171.

10. Kilani M, Micallef J, Soubrouillard C, et al. A longitudinal study of the evolution of cognitive function and affective state in patients with amyotrophic lateral sclerosis. Amyotroph Lateral Scler Other Motor Neuron Disord 2004;5:46-54.

11. Beeldman E, Govaarts R, de Visser M, et al. Progression of cognitive and behavioural impairment in early amyotrophic lateral sclerosis. J Neurol Neurosurg Psychiatry 2020;91:779780.

12. Castelnovo V, Canu E, Riva N, et al. Progression of cognitive and behavioral disturbances in motor neuron diseases assessed using standard and computer-based batteries. Amyotroph Lateral Scler Frontotemporal Degener 2021:1-14.

13. Seeley WW, Crawford R, Rascovsky K, et al. Frontal paralimbic network atrophy in very mild behavioral variant frontotemporal dementia. Arch Neurol 2008;65:249-255.

14. Whitwell JL, Jack CR, Jr., Parisi JE, et al. Imaging signatures of molecular pathology in behavioral variant frontotemporal dementia. J Mol Neurosci 2011;45:372-378.

15. Trojsi F, Esposito F, de Stefano M, et al. Functional overlap and divergence between ALS and bvFTD. Neurobiol Aging 2015;36:413-423.

16. Gordon E, Rohrer JD, Fox NC. Advances in neuroimaging in frontotemporal dementia. J Neurochem 2016;138 Suppl 1:193-210.

17. Agosta F, Sala S, Valsasina P, et al. Brain network connectivity assessed using graph theory in frontotemporal dementia. Neurology 2013;81:134-143.

18. Filippi M, Basaia S, Canu E, et al. Brain network connectivity differs in early-onset neurodegenerative dementia. Neurology 2017;89:1764-1772.

19. Agosta F, Ferraro PM, Riva N, et al. Structural brain correlates of cognitive and behavioral impairment in MND. Hum Brain Mapp 2016;37:1614-1626.

20. Alruwaili AR, Pannek K, Coulthard A, Henderson R, Kurniawan ND, McCombe P. A combined tract-based spatial statistics and voxel-based morphometry study of the first MRI scan 
after diagnosis of amyotrophic lateral sclerosis with subgroup analysis. J Neuroradiol 2018;45:41-48.

21. Illan-Gala I, Montal V, Pegueroles J, et al. Cortical microstructure in the amyotrophic lateral sclerosis-frontotemporal dementia continuum. Neurology 2020;95:e2565-e2576.

22. Kasper E, Schuster C, Machts J, et al. Microstructural white matter changes underlying cognitive and behavioural impairment in ALS--an in vivo study using DTI. PLoS One 2014;9:e114543.

23. van der Burgh HK, Westeneng HJ, Walhout R, et al. Multimodal longitudinal study of structural brain involvement in amyotrophic lateral sclerosis. Neurology 2020;94:e2592-e2604. 24. Schulthess I, Gorges M, Muller HP, et al. Functional connectivity changes resemble patterns of pTDP-43 pathology in amyotrophic lateral sclerosis. Sci Rep 2016;6:38391.

25. Basaia S, Agosta F, Cividini C, et al. Structural and functional brain connectome in motor neuron diseases: A multicenter MRI study. Neurology 2020;95:e2552-e2564.

26. Castelnovo V, Canu E, Calderaro D, et al. Progression of brain functional connectivity and frontal cognitive dysfunction in ALS. Neuroimage Clin 2020;28:102509.

27. Mohammadi B, Kollewe K, Samii A, Krampfl K, Dengler R, Munte TF. Changes of resting state brain networks in amyotrophic lateral sclerosis. Exp Neurol 2009;217:147-153.

28. Hu T, Hou Y, Wei Q, et al. Patterns of brain regional functional coherence in cognitive impaired ALS. Int J Neurosci 2020;130:751-758.

29. Brooks BR, Miller RG, Swash M, Munsat TL, World Federation of Neurology Research Group on Motor Neuron D. El Escorial revisited: revised criteria for the diagnosis of amyotrophic lateral sclerosis. Amyotroph Lateral Scler Other Motor Neuron Disord 2000;1:293299.

30. Rascovsky K, Hodges JR, Knopman D, et al. Sensitivity of revised diagnostic criteria for the behavioural variant of frontotemporal dementia. Brain 2011;134:2456-2477.

31. Cedarbaum JM, Stambler N, Malta E, et al. The ALSFRS-R: a revised ALS functional rating scale that incorporates assessments of respiratory function. BDNF ALS Study Group (Phase III). J Neurol Sci 1999;169:13-21.

32. Knopman DS, Kramer JH, Boeve BF, et al. Development of methodology for conducting clinical trials in frontotemporal lobar degeneration. Brain 2008;131:2957-2968.

33. Schmidt R, Verstraete E, de Reus MA, Veldink JH, van den Berg LH, van den Heuvel MP. Correlation between structural and functional connectivity impairment in amyotrophic lateral sclerosis. Hum Brain Mapp 2014;35:4386-4395.

34. Zalesky A, Fornito A, Bullmore ET. Network-based statistic: identifying differences in brain networks. Neuroimage 2010;53:1197-1207.

35. Pandya S, Kuceyeski A, Raj A, Alzheimer's Disease Neuroimaging I. The Brain's Structural Connectome Mediates the Relationship between Regional Neuroimaging Biomarkers in Alzheimer's Disease. J Alzheimers Dis 2017;55:1639-1657.

36. Reyes P, Ortega-Merchan MP, Rueda A, et al. Functional Connectivity Changes in Behavioral, Semantic, and Nonfluent Variants of Frontotemporal Dementia. Behav Neurol 2018;2018:9684129.

37. Muller HP, Lule D, Roselli F, Behler A, Ludolph AC, Kassubek J. Segmental involvement of the corpus callosum in C9orf72-associated ALS: a tract of interest-based DTI study. Ther Adv Chronic Dis 2021;12:20406223211002969.

38. Tu S, Menke RAL, Talbot K, Kiernan MC, Turner MR. Regional thalamic MRI as a marker of widespread cortical pathology and progressive frontotemporal involvement in amyotrophic lateral sclerosis. J Neurol Neurosurg Psychiatry 2018;89:1250-1258.

39. Meier JM, van der Burgh HK, Nitert AD, et al. Connectome-Based Propagation Model in Amyotrophic Lateral Sclerosis. Ann Neurol 2020;87:725-738.

40. Agosta F, Canu E, Valsasina P, et al. Divergent brain network connectivity in amyotrophic lateral sclerosis. Neurobiol Aging 2013;34:419-427. 
41. Bede P, Omer T, Finegan E, et al. Connectivity-based characterisation of subcortical grey matter pathology in frontotemporal dementia and ALS: a multimodal neuroimaging study. Brain Imaging Behav 2018;12:1696-1707.

42. Menke RAL, Proudfoot M, Talbot K, Turner MR. The two-year progression of structural and functional cerebral MRI in amyotrophic lateral sclerosis. Neuroimage Clin 2018;17:953-961. 43. Van den Bos MAJ, Higashihara M, Geevasinga N, Menon P, Kiernan MC, Vucic S. Imbalance of cortical facilitatory and inhibitory circuits underlies hyperexcitability in ALS. Neurology 2018;91:e1669-e1676.

44. Crabe R, Aimond F, Gosset P, Scamps F, Raoul C. How Degeneration of Cells Surrounding Motoneurons Contributes to Amyotrophic Lateral Sclerosis. Cells 2020;9. 45. Costello E, Rooney J, Pinto-Grau M, et al. Cognitive reserve in amyotrophic lateral sclerosis (ALS): a population-based longitudinal study. J Neurol Neurosurg Psychiatry 2021. 46. Saxon JA, Thompson JC, Harris JM, et al. Cognition and behaviour in frontotemporal dementia with and without amyotrophic lateral sclerosis. J Neurol Neurosurg Psychiatry 2020;91:1304-1311.

47. Canosa A, Moglia C, Manera U, et al. Metabolic brain changes across different levels of cognitive impairment in ALS: a (18)F-FDG-PET study. J Neurol Neurosurg Psychiatry 2020.

48. Braak H, Brettschneider J, Ludolph AC, Lee VM, Trojanowski JQ, Del Tredici K. Amyotrophic lateral sclerosis--a model of corticofugal axonal spread. Nat Rev Neurol 2013;9:708-714.

49. Rohrer JD, Geser F, Zhou J, et al. TDP-43 subtypes are associated with distinct atrophy patterns in frontotemporal dementia. Neurology 2010;75:2204-2211.

50. Omer T, Finegan E, Hutchinson S, et al. Neuroimaging patterns along the ALS-FTD spectrum: a multiparametric imaging study. Amyotroph Lateral Scler Frontotemporal Degener 2017;18:611-623. 
Table 1. Demographic and clinical features of healthy controls, bvFTD patients and ALS patient groups.

\begin{tabular}{|c|c|c|c|c|c|}
\hline & $\begin{array}{l}\text { Healthy } \\
\text { controls }\end{array}$ & ALS-cn & ALS-ci/bi & ALS-FTD & bvFTD \\
\hline $\mathbf{N}$ & 61 & 54 & 21 & 8 & 35 \\
\hline $\begin{array}{c}\text { Age } \\
\text { [years] }\end{array}$ & $\begin{array}{c}63.04 \pm 8.46 \\
(43.36-81.81)\end{array}$ & $\begin{array}{c}61.08 \pm 9.96 \\
(36.38-81.26)\end{array}$ & $\begin{array}{l}67.99 \pm 11.77^{\wedge} \\
(39.89-86.12)\end{array}$ & $\begin{array}{c}60.28 \pm 10.54 \\
(44.68-70.06)\end{array}$ & $\begin{array}{c}63.18 \pm 9.13 \\
(45.51-74.83)\end{array}$ \\
\hline $\begin{array}{c}\text { Sex } \\
\text { [women/men] }\end{array}$ & $36 / 25$ & $19 / 35^{*}$ & $11 / 10$ & & $12 / 23 *$ \\
\hline $\begin{array}{l}\text { Education } \\
\text { [years] }\end{array}$ & $\begin{array}{c}12.89 \pm 4.79 \\
(5.00-24.00)\end{array}$ & $\begin{array}{c}11.06 \pm 4.52 \\
(5.00-24.00)\end{array}$ & $\begin{array}{c}8.38 \pm 3.72 * \\
(3.00-18.00)\end{array}$ & $\begin{array}{l}11.50 \pm 5.95 \\
(4.00-18.00)\end{array}$ & $\begin{array}{c}9.56 \pm 3.65^{*} \\
(4.00-17.00)\end{array}$ \\
\hline $\begin{array}{c}\text { Onset } \\
\text { [limb/bulbar/ } \\
\text { limb+bulbar] }\end{array}$ & - & $41 / 12 / 1$ & $16 / 5 / 0$ & $3 / 5 / 0$ & - \\
\hline $\begin{array}{c}\text { Disease } \\
\text { duration } \\
\text { [months] }\end{array}$ & - & $\begin{array}{l}23.76 \pm 23.96 \# \\
(4.00-136.00)\end{array}$ & $\begin{array}{c}16.62 \pm 12.11 \# \\
(4.00-47.00)\end{array}$ & $\begin{array}{l}23.25 \pm 17.06 \\
(7.00-56.00)\end{array}$ & $\begin{array}{c}41.00 \pm 29.63 \\
(6.87-144.70)\end{array}$ \\
\hline $\begin{array}{c}\text { ALSFRS-r } \\
{[0-48]}\end{array}$ & - & $\begin{array}{c}38.31 \pm 5.46 \\
(23.00-47.00)\end{array}$ & $\begin{array}{c}39.00 \pm 5.72 \\
(28.00-46.00)\end{array}$ & $\begin{array}{c}35.63 \pm 7.84 \\
(24.00-45.00)\end{array}$ & - \\
\hline UMN score & & $\begin{array}{c}11.22 \pm 4.39 \\
(0.00-16.00)\end{array}$ & $\begin{array}{c}10.45 \pm 3.78 \\
(2.00-16.00)\end{array}$ & $\begin{array}{c}12.67 \pm 5.47 \\
(2.00-16.00)\end{array}$ & - \\
\hline $\begin{array}{l}\text { MRC global } \\
\text { score }\end{array}$ & & $\begin{array}{c}102.94 \pm 15.39 \\
(60.00-148.00)\end{array}$ & $\begin{array}{c}101.20 \pm 17.62 \\
(71.00-127.00)\end{array}$ & $\begin{array}{c}108.17 \pm 8.33 \\
(98.00-118)\end{array}$ & - \\
\hline $\begin{array}{c}\text { Disease } \\
\text { progression rate }\end{array}$ & - & $\begin{array}{c}0.64 \pm 0.56 \\
(0.04-2.67)\end{array}$ & $\begin{array}{c}0.77 \pm 0.67 \\
(0.13-2.86)\end{array}$ & $\begin{array}{c}0.57 \pm 0.22 \\
(0.33-1.00)\end{array}$ & - \\
\hline ADL & & - & - & - & $\begin{array}{c}5.62 \pm 0.85 \\
(2.00-6.00)\end{array}$ \\
\hline IADL & - & - & - & - & $\begin{array}{c}4.77 \pm 2.29 \\
(1.00-8.00)\end{array}$ \\
\hline CDR & - & - & - & - & $\begin{array}{c}0.96 \pm 0.57 \\
(0.50-2.00)\end{array}$ \\
\hline CDR SB & - & - & - & - & $\begin{array}{c}4.81 \pm 2.53 \\
(1.00-9.50)\end{array}$ \\
\hline
\end{tabular}

Values are numbers or means \pm standard deviations (range). Disease duration was defined as months from onset to date of MRI scan. The rate of disease progression in ALS patients was defined as follows: (48-ALSFRS-r score)/time from symptom onset. P values refer to ANOVA models, followed by post-hoc pairwise comparisons (Bonferroni-corrected for multiple comparisons), or Chi-squared test. *: $\mathrm{p}<0.05$ vs HC; \#: $\mathrm{p}<0.05$ vs bvFTD; ${ }^{\wedge}$ : $\mathrm{p}<0.05$ vs ALS-cn. 
Abbreviations: ADL= Activities of Daily Living; ALS-ci/bi= ALS with cognitive and/or behavioral impairment; ALS-FTD= ALS with Frontotemporal Dementia; ALS-cn= Amyotrophic lateral sclerosis with only motor impairment; ALSFRS-r= Amyotrophic lateral sclerosis functional rating scale revised; bvFTD = behavioral variant of Frontotemporal Dementia; $\mathrm{CDR}=$ Clinical dementia rating; $\mathrm{CDR} \mathrm{sb}=\mathrm{Clinical}$ dementia rating sum of boxes; $\mathrm{HC}=$ healthy controls; $\mathrm{IADL}=$ Instrumental Activities of Daily Living; MRC = Medical Research Council; $\mathrm{N}=$ Number; UMN= Upper motor neuron. 
Table 2. Classification (ROC curves) analysis for identification of the "ALS-cn-like pattern" and the "bvFTD-like pattern".

\begin{tabular}{|l|c|c|c|}
\hline \multicolumn{4}{|c|}{ Structural Connectivity } \\
\hline $\begin{array}{c}\text { Intra-area and Inter-areas } \\
\text { connections }\end{array}$ & $\begin{array}{c}\text { AUC } \\
\text { bvFTD vs ALS-cn }\end{array}$ & $\begin{array}{c}\text { AUC } \\
\text { ALS-cn } \text { vs bvFTD }\end{array}$ & $\begin{array}{c}\text { Best Cut-off } \\
\text { (Youden's index) }\end{array}$ \\
\hline Frontal & $\mathbf{0 . 8 8}$ & 0.12 & $-0.21(0.68)$ \\
\hline Frontal-Basal Ganglia & $\mathbf{0 . 8 2}$ & 0.18 & $-0.67(0.52)$ \\
\hline Frontal-Parietal & $\mathbf{0 . 7 1}$ & 0.29 & $-0.30(0.37)$ \\
\hline Frontal-Temporal & $\mathbf{0 . 7 6}$ & 0.24 & $-0.20(0.44)$ \\
\hline Sensorimotor-Basal Ganglia & 0.33 & $\mathbf{0 . 6 7}$ & $-0.55(0.30)$ \\
\hline Basal Ganglia-Temporal & $\mathbf{0 . 7 8}$ & 0.22 & $-0.52(0.45)$ \\
\hline Basal Ganglia-Occipital & $\mathbf{0 . 7 2}$ & 0.28 & $-0.47(0.42)$ \\
\hline Parietal & $\mathbf{0 . 6 7}$ & 0.33 & $0.003(0.31)$ \\
\hline Parietal-Temporal & $\mathbf{0 . 7 2}$ & 0.28 & $-0.36(0.43)$ \\
\hline Parietal-Occipital & $\mathbf{0 . 6 9}$ & 0.31 & $-0.08(0.35)$ \\
\hline Temporal & $\mathbf{0 . 7 5}$ & 0.25 & $-0.2(0.41)$ \\
\hline Temporal-Occipital & $\mathbf{0 . 7 0}$ & 0.30 & $-0.40(0.35)$ \\
\hline & Functional Connectivity & & \\
\hline Frontal-Temporal & $\mathbf{0 . 7 7}$ & 0.23 & $-0.27(0.48)$ \\
\hline Sensorimotor-Parietal & $\mathbf{0 . 6 7}$ & 0.33 & $-0.04(0.34)$ \\
\hline
\end{tabular}

The Area Under the ROC curve represents the capability of the structural and functional connectivity damage within the reported intra- and inter-areas to discriminate bvFTD from ALS-cn and viceversa. Only intra-area and inter-areas connections significantly different between the two groups were considered. Bold values in the column "bvFTD vs ALS-cn" identify the "bvFTD-like pattern". Bold values in the column "ALS-cn vs bvFTD" identify the "ALS-cn-like pattern". The optimal cut-off per each connectivity distribution was calculated through the Youden's index, maximizing sensibility and specificity. Cut-off $=$ sensibility-(1-specificity). Abbreviations: ALS-cn= Amyotrophic lateral sclerosis with only motor impairment; AUC= Area Under the ROC curve; bvFTD= behavioral variant of Frontotemporal Dementia. 


\section{Figure legends}

Figure 1. Study Framework. (A) Patient classification. Revised Strong's Criteria were applied to identify ALS patients with and without cognitive/behavioral impairments or dementia deficits. (B)

Connectome Reconstruction. Connectomics was apply on DT MRI and RS fMRI, after parcellating the brain into 220 regions. Structural and functional connectomes of all subjects were reconstructed. (C) Regional connectivity analysis. The Network based Statistics was performed performing all possible comparisons between groups. (D) Distribution analysis. After reconstructing the structural and functional connectome of each patient and control of the study, all connections per each patient were normalized relative to controls and grouped into 6 macro-areas. Intra-area and inter-area connectivity distribution were plotted and statistically compared between groups. (E) Classification analysis. ROC curve analysis was performed to discriminate ALS-cn from bvFTD and vice versa, considering intra and inter-area connectivity that resulted significantly different between these two groups in the distribution analysis. (F) Frequency analysis. After ROC curve analysis, the optimal cut-off was identified using the Youden's index. ALS-ci/bi and ALS-FTD cases were then subdivided in those under and above the optimal cutoff. Chi-squared test was performed in order to identify the behavior of these two groups. Abbreviations: ALS= Amyotrophic lateral sclerosis;

ALS-ci/bi= ALS with cognitive and/or behavioral impairment; ALS-cn= ALS with motor impairment only; ALS-FTD= ALS with Frontotemporal Dementia; bvFTD= behavioral variant of Frontotemporal Dementia; DT MRI= diffusion tensor MRI; fMRI= functional MRI; HC= healthy controls; SBJ= subject. 


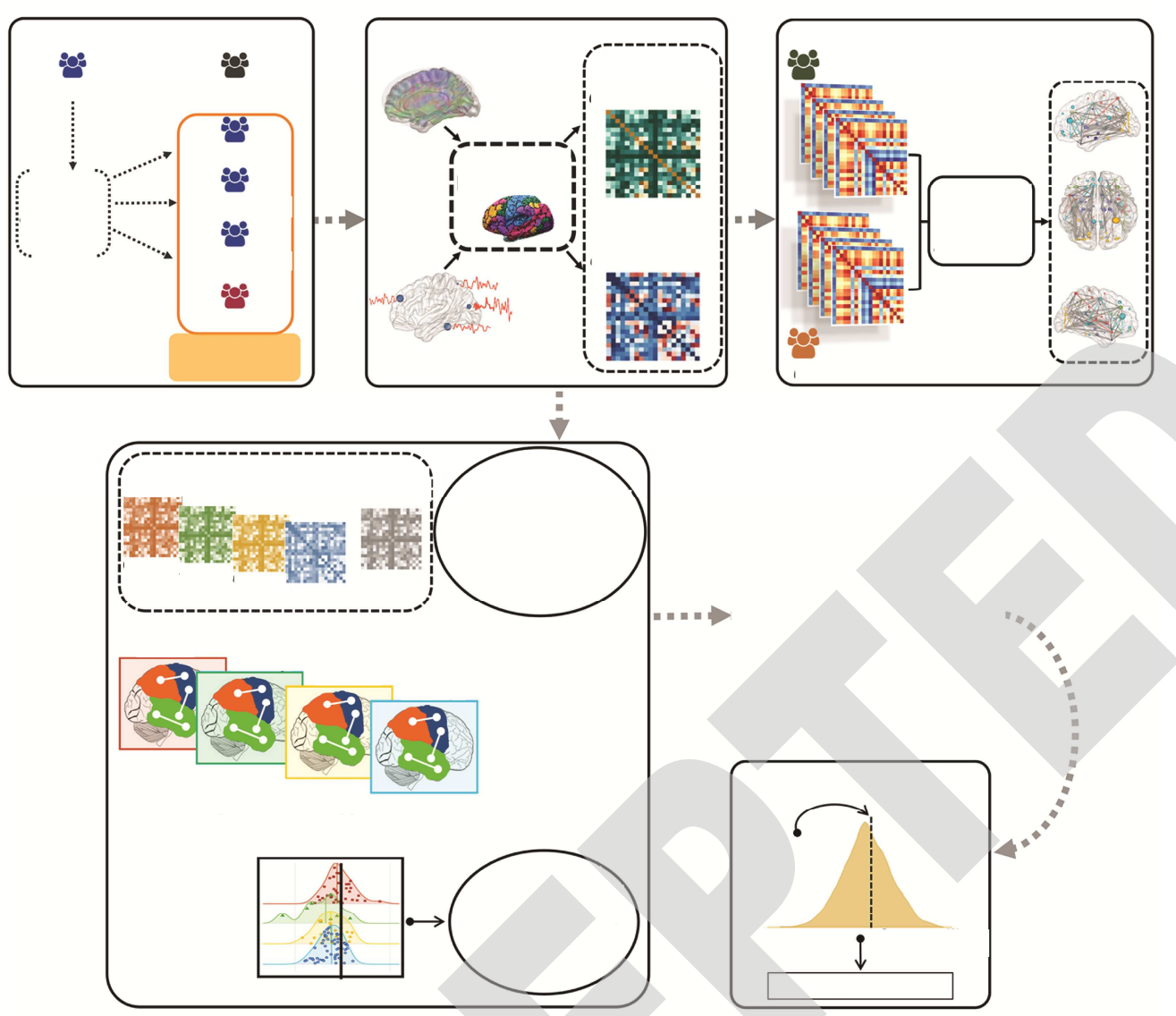


Figure 2. Alterations in structural and functional connectivity in ALS and bvFTD patients relative to healthy controls and each other. Altered structural (A) and functional (B) connections are represented per each significant contrast, respectively $(\mathrm{p}<0.05)$. The comparisons were adjusted for age, sex and education. The node color represents its belonging to specific macro-areas (frontal, sensorimotor, basal ganglia, parietal, temporal and occipital). The node size is proportional to the number of affected connections (the higher the number of disrupted connections, the bigger the node). Abbreviations: $A=$ anterior; ALS= Amyotrophic lateral sclerosis; ALS-ci/bi= ALS with cognitive and/or behavioral impairment; ALS-cn= ALS with motor impairment only; ALS-FTD= ALS with Frontotemporal Dementia; bvFTD= behavioral variant of Frontotemporal Dementia; FA= fractional anisotropy; $\mathrm{HC}=$ healthy controls; $\mathrm{L}=$ left; $\mathrm{P}=$ posterior; $\mathrm{R}=$ right. 
A. Structural (FA)

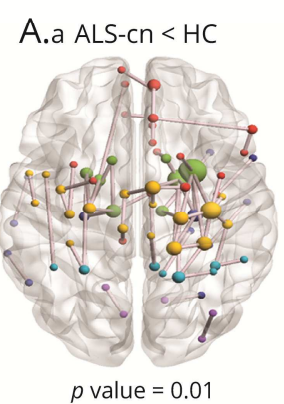

A.e ALS-cn < bvFTD

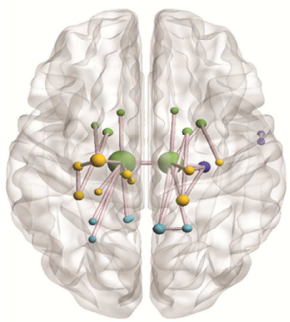

$p$ value $=0.03$

B. Functional
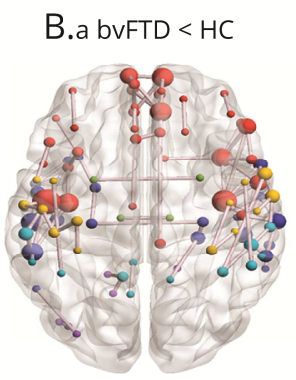

$p$ value $=0.02$

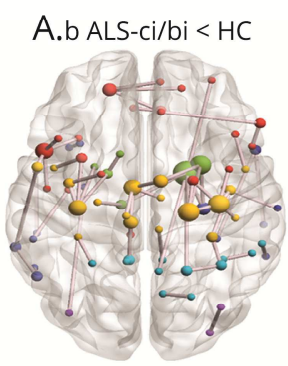

$p$ value $=0.02$

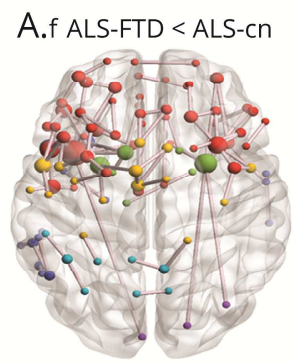

$p$ value $=0.01$

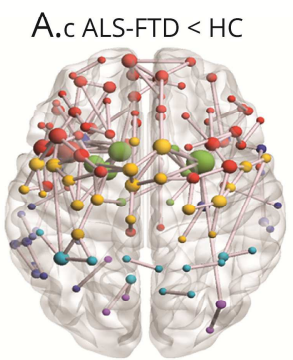

$p$ value $=0.001$

A.g bvFTD < ALS-cn

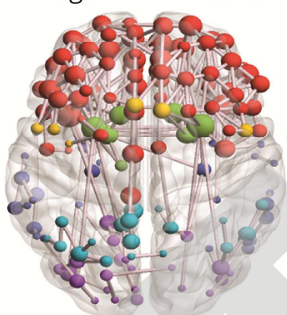

$p$ value $<0.001$

B. c bvFTD $<$ ALS-cn

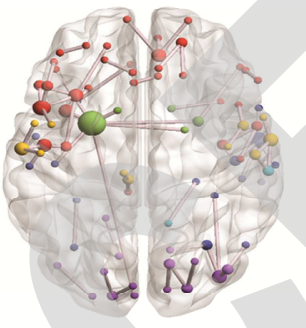

$p$ value $=0.02$

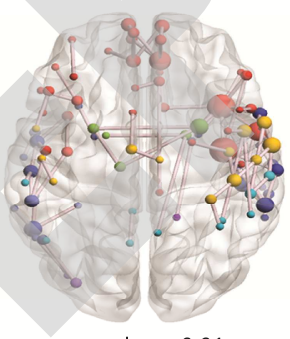

$p$ value $<0.01$

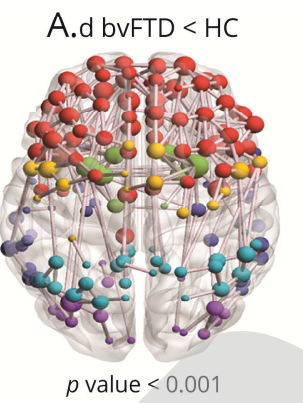

A.h bvFTD $<$ ALS-ci/bi

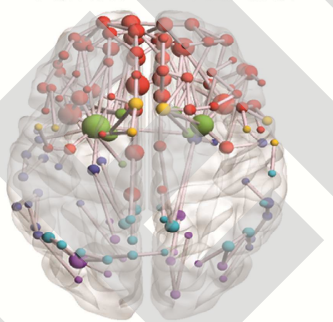

$p$ value $<0.001$

B.d bvFTD $<$ ALS-ci/bi

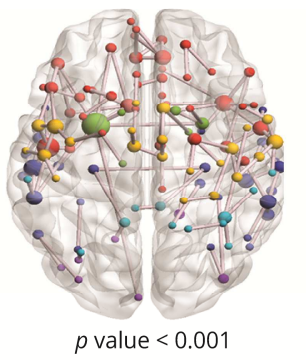

Lobes

Frontal

Sensorimotor

Basal-yanly
Parietal

Temporal

Occipital

Node size

00

Proportional to the number of affected connections

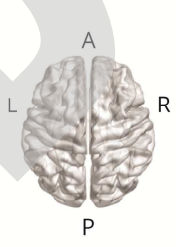

Lobes

Frontal

Sensorimotor Basal-ganglia Parietal

Temporal

Occipital

Node size

$\mathrm{OOO}$

Proportional to the number of affected connections 
Figure 3. Distribution analysis of the structural connectivity damage in patient groups. The distribution of the structural connectivity alterations within frontal and motor areas and in the connections towards these areas is displayed. Distribution curves are normalized relative to control values. The more the curve is shifted towards negative values, the greater is the structural damage. All significant contrasts $(\mathrm{p}<0.05)$ - displayed with colored stars - are reported according to age-, sex- and education-adjusted ANOVA models, Bonferroni-corrected for multiple comparisons. Abbreviations: ALS= Amyotrophic lateral sclerosis; ALS-ci/bi= ALS with cognitive and/or behavioral impairment; ALS-cn= ALS with motor impairment only; ALS-FTD= ALS with Frontotemporal Dementia; bvFTD= behavioral variant of Frontotemporal Dementia. 


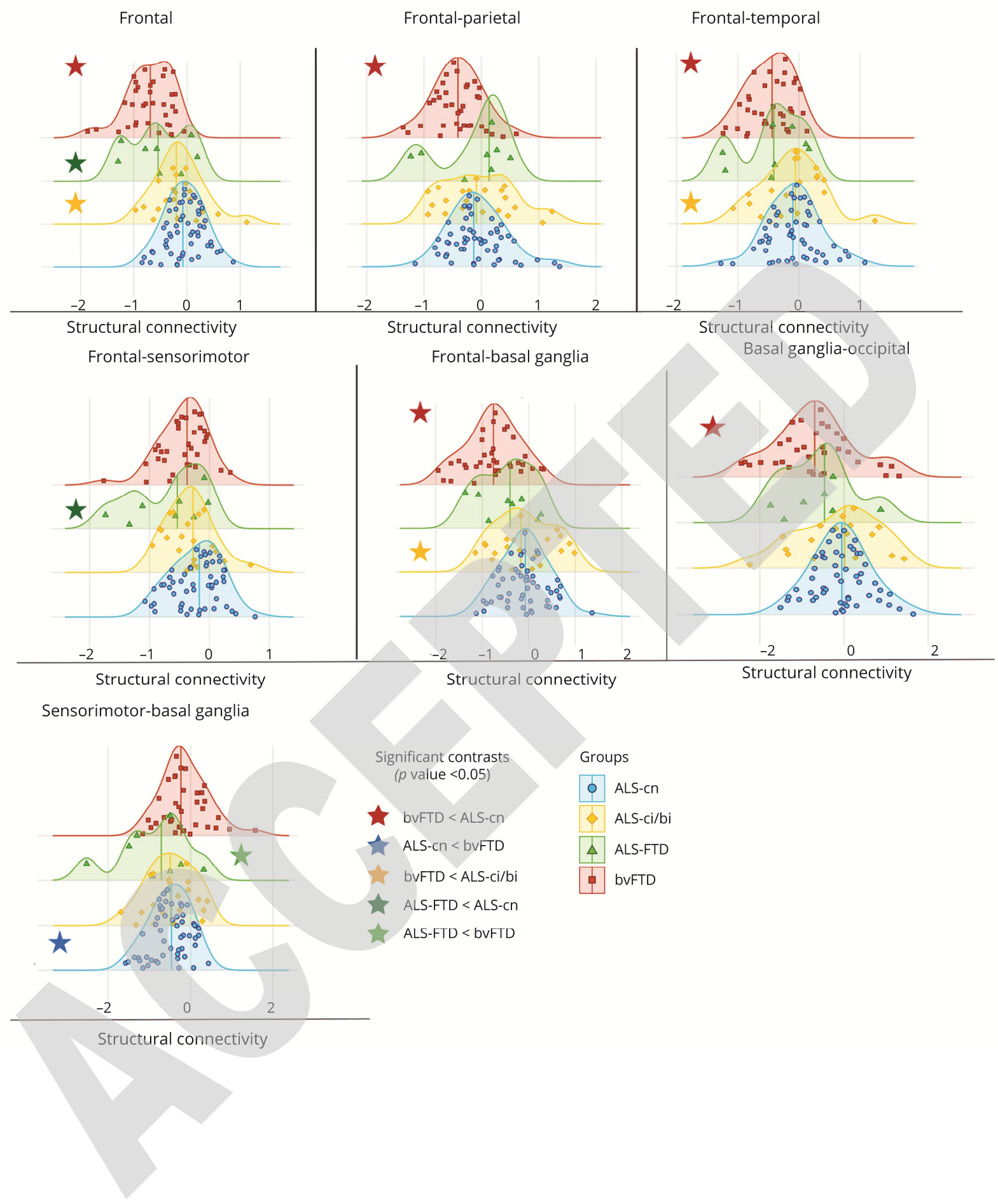


Figure 4. Distribution analysis of the structural connectivity damage in patient groups. The distribution of the structural connectivity alterations within parietal and temporal areas and in the connections towards these areas is displayed. Distribution curves are normalized relative to control values. The more the curve is shifted towards negative values, the greater is the structural damage. All significant contrasts $(\mathrm{p}<0.05)$ - displayed with colored stars - are reported according to age-, sex- and education-adjusted ANOVA models, Bonferroni-corrected for multiple comparisons. Abbreviations: ALS= Amyotrophic lateral sclerosis; ALS-ci/bi= ALS with cognitive and/or behavioral impairment; ALS-cn= ALS with motor impairment only; ALS-FTD= ALS with Frontotemporal Dementia; bvFTD= behavioral variant of Frontotemporal Dementia.

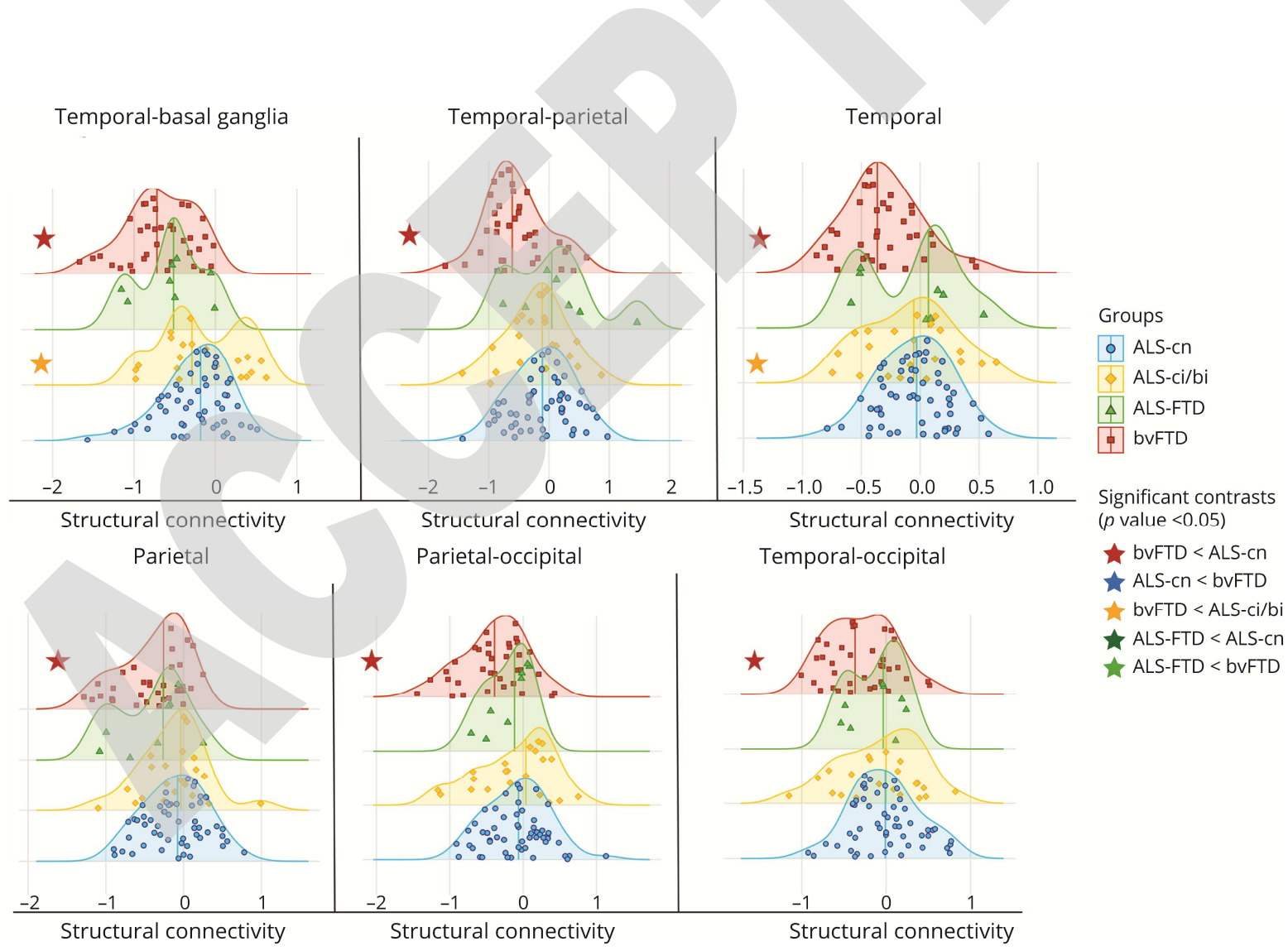


Figure 5. Distribution analysis of the functional connectivity damage in patient groups.

Functional connectivity damage distribution within area and among areas is reported. Distribution curves are normalized relative to control values. The more the curve is shifted towards negative values, the more reduced is the functional connectivity. All significant contrasts $(p<0.05)-$ displayed with colored stars - are reported according to age-, sex- and education-adjusted ANOVA models, Bonferroni-corrected for multiple comparisons. Abbreviations: ALS= Amyotrophic lateral sclerosis; ALS-ci/bi= ALS with cognitive and/or behavioral impairment; ALS-cn= ALS with motor impairment only; ALS-FTD= ALS with Frontotemporal Dementia; bvFTD= behavioral variant of Frontotemporal Dementia.

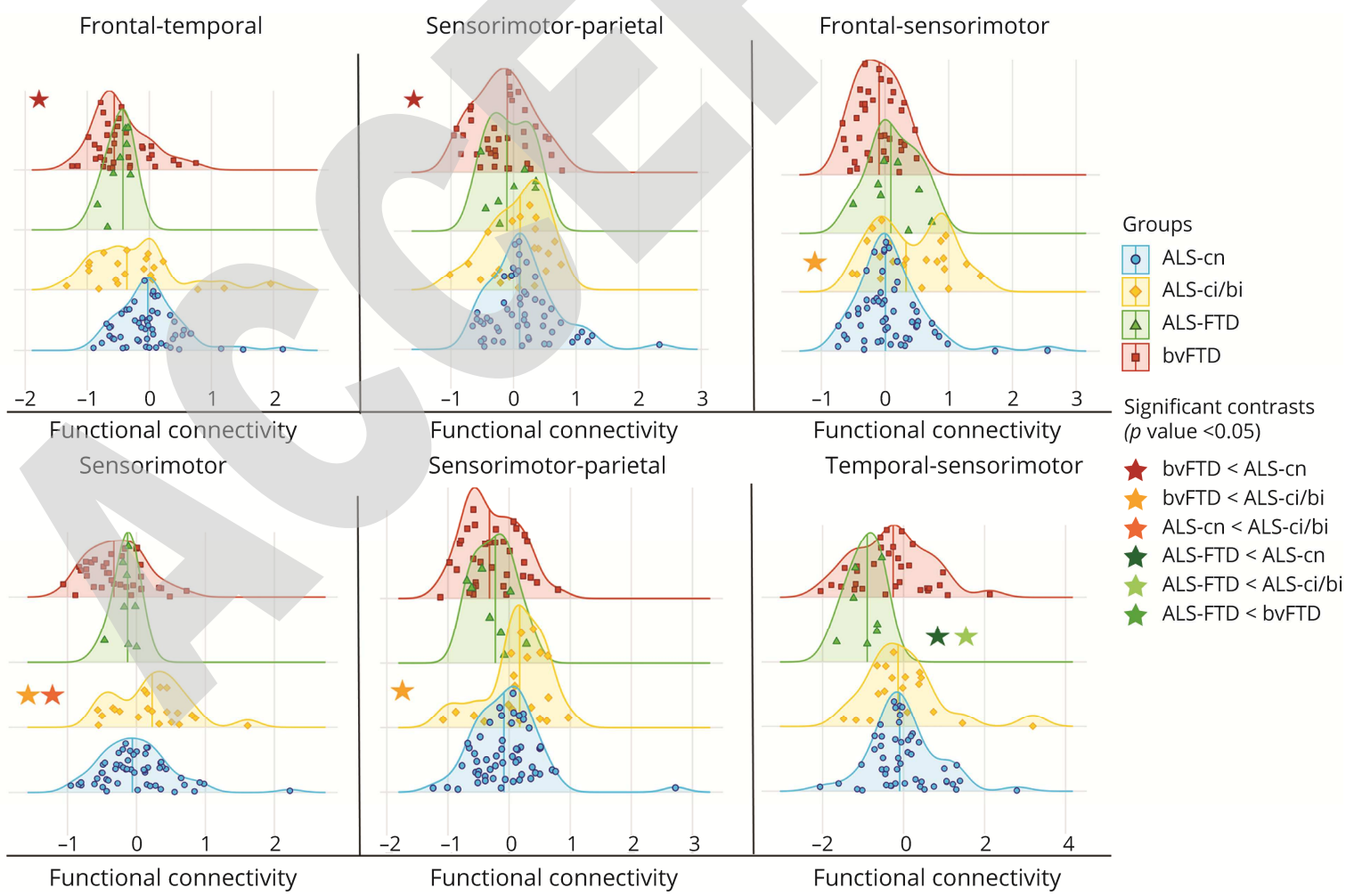




\title{
Neurology
}

\author{
Amyotrophic Lateral Sclerosis-Frontotemporal Dementia: Shared and Divergent Neural \\ Correlates Across the Clinical Spectrum \\ Camilla Cividini, Silvia Basaia, Edoardo G. Spinelli, et al. \\ Neurology published online December 1, 2021 \\ DOI 10.1212/WNL.0000000000013123
}

This information is current as of December 1, 2021

Updated Information \&

Services

Subspecialty Collections

Permissions \& Licensing

Reprints including high resolution figures, can be found at:

http://n.neurology.org/content/early/2021/12/01/WNL.0000000000013123.f ull

This article, along with others on similar topics, appears in the following collection(s):

Amyotrophic lateral sclerosis

http://n.neurology.org/cgi/collection/amyotrophic_lateral_sclerosis_ fMRI

http://n.neurology.org/cgi/collection/fmri

Frontotemporal dementia

http://n.neurology.org/cgi/collection/frontotemporal_dementia

Information about reproducing this article in parts (figures,tables) or in its entirety can be found online at:

http://www.neurology.org/about/about_the_journal\#permissions

Information about ordering reprints can be found online:

http://n.neurology.org/subscribers/advertise

Neurology $®$ is the official journal of the American Academy of Neurology. Published continuously since 1951, it is now a weekly with 48 issues per year. Copyright Copyright (C) 2021 The Author(s). Published by Wolters

Kluwer Health, Inc. on behalf of the American Academy of Neurology.. All rights reserved. Print ISSN:

0028-3878. Online ISSN: 1526-632X.

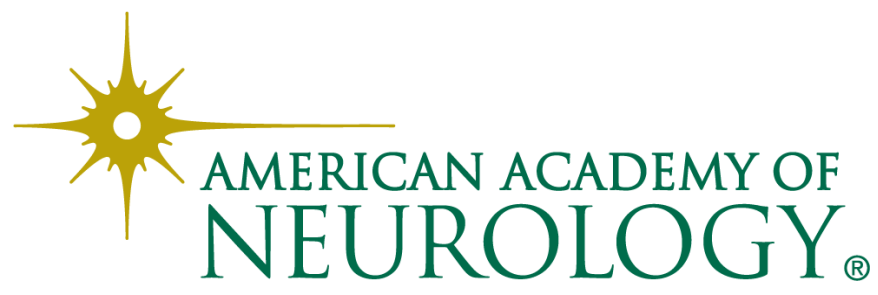

